\title{
Uso de cateteres venosos totalmente implantados para nutrição parenteral: cuidados, tempo de permanência e ocorrência de complicações infecciosas
}

\author{
Long-term central venous catheter for total \\ parenteral nutrition: Catheter care, permanence \\ period, and incidence of infections
}

Maria do Rosário Del Lama de UNAMUNO ${ }^{1}$

João José CARNEIRO²

Fernando Bahdur CHUEIRE ${ }^{1}$

Júlio Sérgio MARCHINI ${ }^{1}$

Vivian Marques Miguel SUEN' ${ }^{1}$

Cateteres venosos totalmente implantados são utilizados em pacientes com síndrome do intestino curto, para realizar o suporte nutricional parenteral, o qual mantém estes pacientes vivos, pois fornece-lhes nutrientes que são absorvidos pela via digestiva. No entanto, estes cateteres não são isentos de complicações. As infecções relacionadas aos cateteres venosos são as complicações mais temidas e sua incidência varia de 3\% a $20 \%$, aumentando em pacientes mais graves. O objetivo do presente estudo é descrever as complicações infecciosas em pacientes recebendo nutrição parenteral por meio de cateteres venosos totalmente implantados. Tais cateteres são utilizados pela Divisão de Nutrição Clínica do Hospital das Clínicas da Faculdade de Medicina de Ribeirão Preto, Universidade de São Paulo, para realizar o suporte nutricional parenteral em pacientes submetidos a ressecções extensas de intestino delgado. Foram avaliadas as complicações infecciosas ocorridas com 21 cateteres, implantados em 16 pacientes. O tempo de permanência dos cateteres foi de 768 $\pm 664,3$ dias (mediana 529 dias) e a taxa de infecção foi de 0,029 infecções/paciente/ano, resultados que se comparam às taxas de infecção observadas em países desenvolvidos. Concluiu-se que os cuidados observados no manuseio destes cateteres foram de fundamental importância para diminuir a incidência de infecção nestes pacientes.

Termos de indexação: cateter, nutrição parenteral, sepse.

\footnotetext{
1 Hospital das Clínicas, Faculdade de Medicina de Ribeirão Preto, Divisão de Nutrição Clínica, Unidade Metabólica, Universidade de São Paulo. Av. Bandeirantes, 3900, 5aandar, 14048-900, Ribeirão Preto, SP, Brasil. Correspondência para/Correspondence to: M.R.D.L. UNAMUNO.E-mail: <unamuno@usp.br>.

2 Divisão de Cirurgia Toráxica e Cárdio Vascular, Faculdade de Medicina de Ribeirão Preto, Universidade de São Paulo. São Paulo, SP, Brasil.
} 


\section{A B S T R A C T}

Long-term venous catheters are used for the total parenteral nutrition infusion, which is essential for feeding short-bowel syndrome patients. However, complications are likely to occur. The incidence of catheter related infections ranges from 3 to $20 \%$ in hospitalized patients. The Divisão de Nutrição Clínica do Hospital das Clínicas da Faculdade de Medicina de Ribeirão Preto, University of São Paulo, Brazil, has been providing nutrition support to short-bowel syndrome patients, using totally implantable venous catheters. This is a retrospective study, which aims to show the Divisão de Nutrição Clínica experience with such catheters, and to discuss the incidence of infections. We retrospectively evaluated infection complications in 21 catheters implanted in 16 patients. The permanence-time of the catheters was 768 664.3 days (median: 529 days). The incidence of infection was 0.029 per patient, per year. These results are comparable to data from developed countries. Considering that the patients here studied were imunossupressed due to intestinal mal-absorption, our findings suggest that the catheter care, as provided by our team, contributed to diminish the rate of infections, and to increase the utilization period of each catheter.

Indexing terms: catheters, parenteral nutrition, sepsis.

\section{N T R O D U Ç Ã O}

Pacientes submetidos a ressecções extensas de intestino delgado, inevitavelmente cursam com síndrome de má absorção e, portanto, necessitam receber nutrientes endovenosamente. Em nosso meio, é grande o número de pacientes submetidos a este tipo de cirurgia; por serem situações de extrema urgência e gravidade, $40 \%$ a $80 \%$ dos casos evoluem para óbito, mesmo antes da alta hospitalar, como é mostrado na Figura 1.
Na ausência de suporte nutricional por via endovenosa, a taxa de sobrevida dos pacientes submetidos a ressecções de mais de 2 metros de intestino delgado é de seis meses. Desta maneira, quando encaminhados à Unidade Metabólica do Hospital das Clínicas da Faculdade de Medicina de Ribeirão Preto, da Universidade de São Paulo (HCFMRP-USP), os pacientes enterectomizados recebem um cateter totalmente implantado. Este cateter é vital para a administração de nutrientes por via endovenosa, por tempo indeterminado ou até definitivamente, e tem contribuído para a

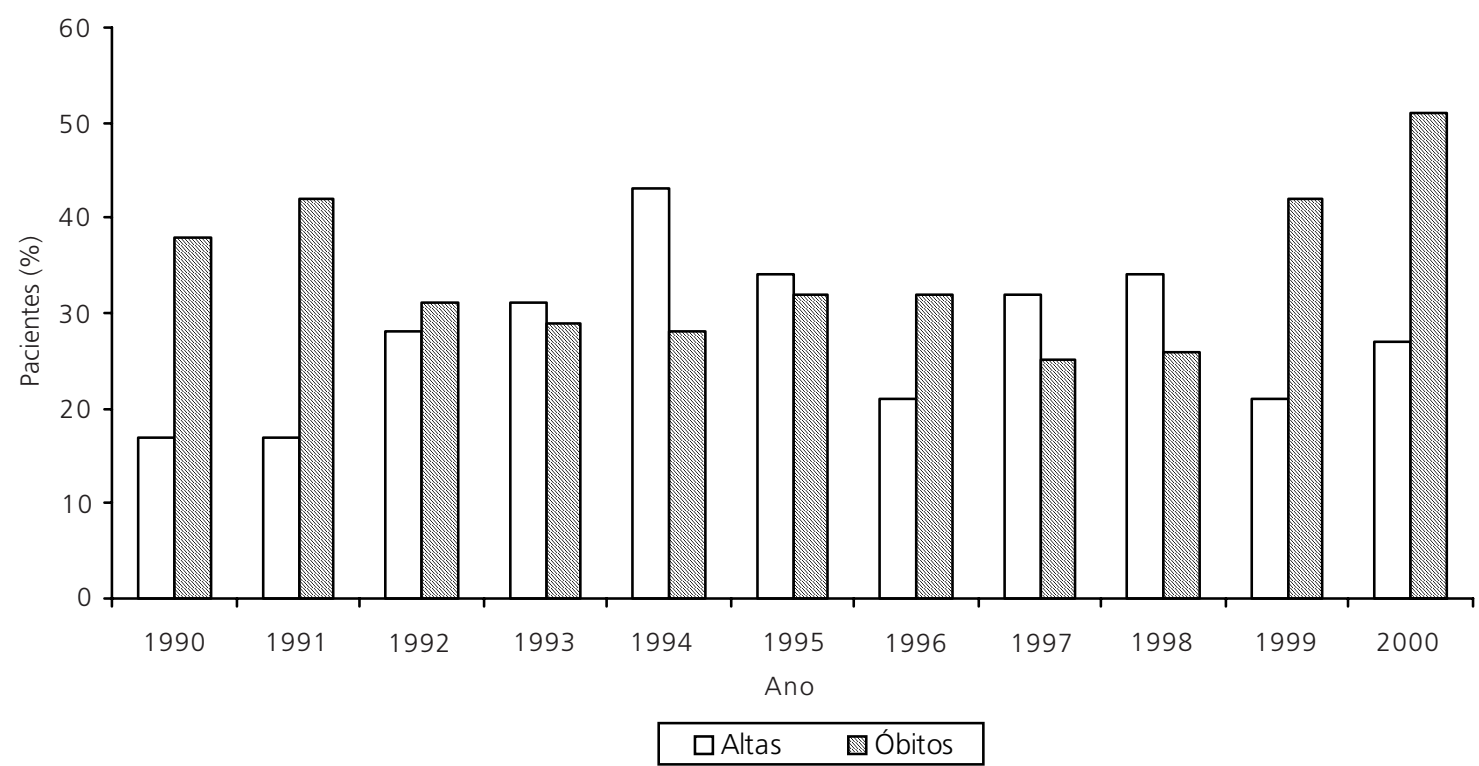

Figura 1. Pacientes que evoluíram para óbito e os que receberam alta hospitalar, após ressecção de intestino delgado. Unidade de Emergência do HCFMRP-USP, 1990 a 2000. 
prevenção da subnutrição e da evolução para o óbito.

$\mathrm{Na}$ década de 80, os cateteres com reservatórios ou "Port" foram projetados por Neiderhuber et al. ${ }^{1,2}$ para pacientes oncológicos. São implantados cirurgicamente e apresentam durabilidade de vários anos. Devido ao seu dispositivo de silicone polimerizável, suportam cerca de 2000 punções com agulhas curvas especiais, que mantêm a integridade de sua estrutura. A implantação deste cateter em pacientes desnutridos é feita sob anestesia local e subpeitoral ${ }^{3}$. Anualmente, cerca de 500 mil cateteres venosos centrais de longa permanência são implantados nos Estados Unidos para quimioterapia e nutrição parenteral ${ }^{4}$ e 180 mil infecções relacionadas a cateteres acontecem a cada ano. A incidência de infeções relacionadas a cateteres venosos centrais varia de $3 \%$ a $20 \%$, em pacientes hospitalizados, e estas taxas aumentam em casos mais graves. Os cateteres totalmente implantados determinam menor risco de infecções, quando comparados aos cateteres com extremidade exteriorizada $a^{3,5-12}$.

A patogênese das infecções de cateteres está relacionada com a deposição de microorganismos no cateter no momento da inserção, com a migração de microorganismos através da pele e ao longo do cateter, com a contaminação da conexão e do líquido de infusão, além do foco de infecção a distância ${ }^{13}$.

O treinamento dos profissionais de enfermagem que manipulam diariamente estes cateteres, e a existência de protocolos rigorosos de cuidados, têm possibilitado a redução nos riscos de complicações infecciosas 5 . O microrganismo mais comum nas infecções relacionadas a cateter é o stafilococus coagulase negativo, Staphylococus epidermidis multi-resistente ${ }^{14,15}$; são muito freqüentes também as contaminações do cateter por Staphylococus aureus e fungos ${ }^{16}$.

Muitas vezes, além do processo infeccioso em si, há formação de trombos ao redor do cateter.
Stillman et al. ${ }^{17}$ mostraram que, em uma série de 94 cateteres, $27 \%$ deles tinham trombos visíveis, apresentando culturas positivas, ao contrário dos que não tinham trombos. Dados semelhantes também são descritos por Raad ${ }^{18,19}$ e Timsit ${ }^{20}$.

Devido à alta incidência e gravidade do quadro infeccioso, nos EUA, o "National Nosocomial Infection Surveillance System (NNIS)" monitoriza todas as infecções em pacientes clínicos e cirúrgicos de unidades de terapia intensiva, tendo demonstrado haver uma média de 4 a 5 infecções sangüíneas relacionadas aos diversos tipos de cateteres venosos, num período de 1 mil dias de uso. Esta taxa se eleva para 14 a 15/1000 em pacientes queimados, com custos de US $\$ 2.800$ a US $\$ 32.500$ por incidência infecciosa ${ }^{14}$.

A Divisão de Nutrologia do HCFMRP-USP recebe pacientes com ressecções intestinais extensas, que resultaram em menos de $1 \mathrm{~m}$ de intestino delgado remanescente, muitos destes tendo apenas 15 a $30 \mathrm{~cm}$ de extensão ${ }^{21}$. Nestes pacientes, são implantados cateteres venosos de longa permanência, a fim de fornecer terapia nutricional parenteral. O presente trabalho tem caráter retrospectivo e seu objetivo é descrever as complicações infecciosas em pacientes recebendo terapia nutricional parenteral, por meio de cateter totalmente implantado, na Divisão de Nutrologia do Hospital das Clínicas de Ribeirão Preto.

\section{M É T O D O S}

Participaram do estudo os 16 pacientes que sofreram ressecção intestinal extensa durante o período de 1992 a 2001, os quais receberam nutrição parenteral através de 21 cateteres, com reservatório de infusão totalmente implantado sob o músculo peitoral.

\section{Descrição dos cuidados dispensados ao acesso do cateter e à administração da solução de nutrição parenteral}

Logo após a implantação, os cateteres foram heparinizados e os pacientes receberam 
profilaticamente cefalotina $(4 \mathrm{~g} / \mathrm{dia})$, por via endovenosa, durante três a quatro dias. A infusão de nutrientes somente foi realizada três semanas após a implantação do cateter, quando os pacientes foram internados e passaram a receber nutrição parenteral por períodos de 10 a 160 horas, em intervalos de sete a vinte e cinco dias $22-25$.

Para a administração da nutrição parenteral pelo cateter venoso, o reservatório era localizado com os dedos polegar e indicador, sendo então realizada a assepsia rigorosa local, com solução iodada alcoólica, de maneira centrífuga, durante 3 minutos. A camada de silicone do reservatório era puncionada usando-se microfusor com agulha hubber (20x20G) angulada/90.

A fixação do microfusor à pele foi feita usando-se fita adesiva impermeável larga $(10 \times 10 \mathrm{~cm})$ para evitar o deslocamento da agulha e infiltração da solução no tecido subcutâneo.

A conexão do microfusor com o equipo de solução foi protegida com gaze embebida em solução anti-séptica iodada trocada a cada 24 horas, juntamente com a primeira solução de nutrição prescrita e todo o sistema de infusão ${ }^{26,27}$.

A equipe de enfermagem foi orientada a fazer assepsia da conexão todas as vezes que o sistema era manipulado27,28. A fixação do microfusor foi feita com fita adesiva impermeável larga, usando a técnica compressiva para evitar deslocamento da agulha e infiltração subcutânea da solução nutritiva, o que implicaria em interrupção da infusão e maior manipulação do cateter para uma nova punção.

As soluções nutritivas foram preparadas na farmácia do hospital, em ambiente adequado às exigências da portaria 272 de $1999^{29}$. Após o preparo, estas soluções foram encaminhadas à Unidade Metabólica e conservadas em geladeira a $4^{\circ} \mathrm{C}$. Para a infusão venosa, as soluções estavam à temperatura ambiente e foram administradas usando-se bombas de infusão, permitindo fluxo de líquido contínuo e programado.

Os pacientes foram orientados quanto aos cuidados adequados de higiene, principalmente das mãos.
Com o objetivo de reduzir a manipulação, evitar formação de trombos e conseqüente obstrução do cateter e crescimento microbiano, a aspiração de sangue através do cateter não foi permitida. Padronizou-se a colheita de culturas sangüíneas, feitas somente quando existisse febre ou outro sinal de infecção ${ }^{18,19}$.

Frente à preocupação com o controle das infecções nestes pacientes, passamos a redobrar os cuidados assépticos, protegendo sempre as conexões com gaze embebida em solução anti-séptica ${ }^{28}$.

Terminado o período programado para a infusão da nutrição parenteral, injetava-se clindamicina (300mg) e solução de heparina (1000UI) no cateter, seguido de retirada do microfusor.

Os pacientes receberam alta hospitalar e seu cateter não foi mais manipulado, até uma próxima internação para nova infusão de nutrição parenteral. Permaneceram em suas casas, recebendo alimentação via oral, com uma dieta fracionada ${ }^{23,30-33}$.

\section{Diagnóstico de Infecção Relacionada ao Cateter}

O diagnóstico de infecção relacionada ao cateter foi feito, baseando-nos em critérios laboratorial e clínico. Considerou-se septicemia quando havia pelo menos duas culturas positivas para o mesmo microorganismo no sangue coletado através de punção do cateter e de veia periférica, associado à presença de sintomatologia sugestiva de infecção sistêmica, como febre e tremores persistentes.

\section{RESULTADOS}

Os resultados estão resumidos na Tabela 1. Os 16 pacientes que sofreram ressecções intestinais extensas tiveram um tempo total de uso dos cateteres de 16133 dias. Os 21 cateteres implantados tiveram um tempo de permanência 


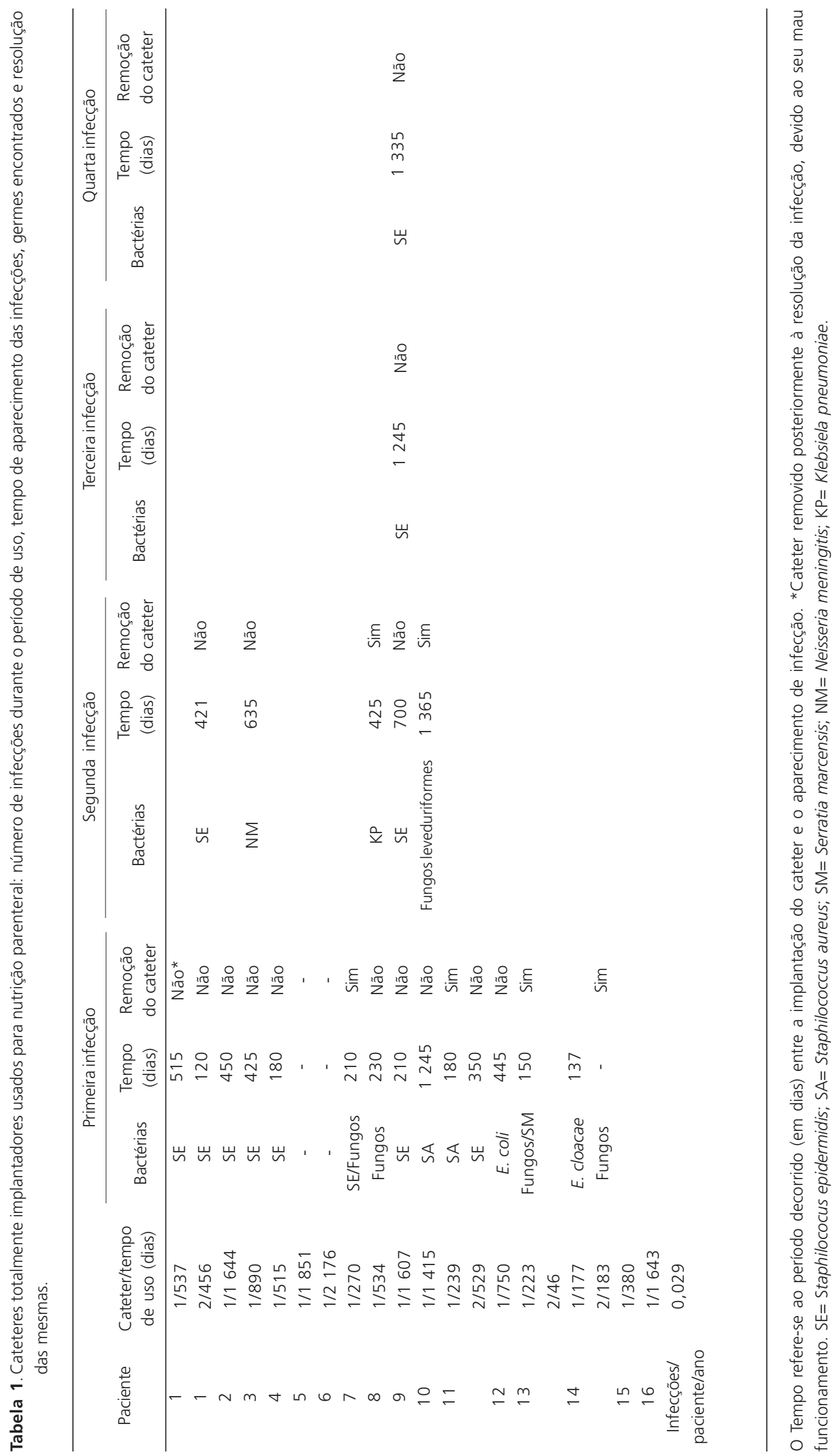


mínimo de 46 dias e máximo de 2173 dias, com tempo médio de uso de 768 1664 dias (mediana 529 dias); a taxa de infecção foi de 0,029 infecções /paciente/ ano.

Neste período de uso, ocorreram 21 episódios de infecções em 14 destes cateteres, com aparecimento da primeira infecção após período que variou de 120 a 1245 dias. Cinco pacientes tiveram uma segunda infecção, que apareceu depois de 210 dias em média, após a primeira infeção. Uma paciente apresentou abcesso na região peri "port", devido a punção por profissional não treinado, resultando na retirada do cateter. Um dos pacientes teve 4 infecções no mesmo cateter, após 210, 700, 1245 e 1335 dias de uso tendo recebido antibioticoterapia (cefalosporina durante 21 dias e amicacina durante 7 dias), administrado através do cateter, durante três semanas. Neste período, foi suspensa a administração da nutrição parenteral. O cateter deste paciente não foi retirado, e no fim do estudo apresentava-se com 1620 dias de uso. Os germes isolados neste paciente em particular apresentaram resistência bacteriana à clindamicina nos dois últimos episódios de infecção.

Do total de 16 pacientes, 3 manifestaram resistência bacteriana à clindamicina, sendo então suspenso o uso deste antibiótico. À presença de infecção por fungos e de abscesso peri-"port", procedia-se à retirada e troca do cateter. Nas demais situações, usou-se antibioticoterapia e o uso do cateter foi continuado. Uma morte foi atribuída à endocardite causada pelo cateter ${ }^{34}$.

\section{I S C U S S Ã O}

Contaminações de cateteres venosos centrais são a principal causa de infecção sangüínea intra-hospitalar e estas infecções são responsáveis pelo aumento da mortalidade destes pacientes ${ }^{18,35}$. Tais infecções geralmente são difíceis de serem diagnosticadas e tratadas, por serem causadas por microorganismos intrahospitalares multiresistentes ${ }^{35,36}$. Desta maneira, a prevenção de infecção é extremamente importante.
O tempo médio de uso dos cateteres aqui estudados foi de $738 \pm 664$ dias, semelhante ou superior aos resultados mostrados em outros centros que também fazem uso deste dispositivo com o objetivo de nutrição e quimioterapia ${ }^{6,12,37-39}$.

O alto custo de técnicas mais sofisticadas usadas para a prevenção de infecção de cateteres venosos, adotadas em outros países, dificulta o uso das mesmas em nosso meio ${ }^{36,40-42}$; portanto, técnicas alternativas com menor custo tornam-se necessárias.

Os resultados aqui apresentados mostram a importância dos cuidados na manipulação destes cateteres em pacientes com síndrome do intestino curto. Observou-se que os cuidados dispensados possibilitaram um longo tempo de permanência dos cateteres, evitando a interrupção da oferta de nutrientes por via parenteral.

Estes cuidados também podem ser responsáveis pela baixa taxa de contaminações destes cateteres $(0,029$ por paciente por ano). Taxa de infecções semelhante à aqui descrita foi encontrada por Young et al..$^{40}$, que reportaram uma taxa média de infecção de 0,020 por paciente por $a^{4} 0^{40}$. Outros estudos encontraram taxas maiores ou menores: O'Keefe et al. descreveram taxa anual de infecções de 0,39 por ano ${ }^{43}$, enquanto Guedon et al. ${ }^{36}$ observaram taxa de infecção de 0,11 por paciente, por ano.

Quanto aos agentes etiológicos das infecções, os microorganismos aqui encontrados também são semelhantes aos microorganismos descritos neste e em outros estudos ${ }^{36,40,44}$. O mais freqüente no presente trabalho foi o Staphylococcus epidermidis (57\%), seguido por fungos, bacilos gram negativos (E coli, Serratia marcescens, Enterobacter Cloacae) e Staphylococcus aureus. A maior incidência de infecções causadas por Staphylococcus epidermidis pode ser explicada pela imunossupressão dos pacientes, que contribui para estas infecções oportunistas. O uso excessivo de antibióticos, levando ao desenvolvimento de cepas resistentes, também pode ser outro fator contribuinte para o desenvolvimento destas infecções. 
Mesmo com a intensa evolução tecnológica, evitar a contaminação, com os cuidados dos cateteres pelos profissionais de saúde, tem importância fundamental na prevenção de infecção e no prolongamento do tempo de permanência dos cateteres totalmente implantados.

Quanto ao diagnóstico dos episódios infecciosos, apesar de cultura quantitativa ser a técnica ideal para o diagnóstico destas infecções, nos casos aqui relatados o diagnóstico foi feito baseado na cultura qualitativa. Há evidências na literatura de que, a cultura positiva do sangue retirado do cateter, associada à cultura positiva do sangue periférico, ambas com o mesmo microor-ganismo, é indicação de infecção relacionada ao cateter ${ }^{45}$.

Considerando-se que os pacientes com síndrome do intestino curto são desnutridos, imuno-deprimidos e dependem do cateter para receber nutrientes de forma adequada, optou-se por tratar com antibioticoterapia os pacientes cujas culturas qualitativas de sangue, de cateter e periférico, mostraram o mesmo microorganismo.

Os resultados aqui apresentados mostram que, apesar da intensa evolução tecnológica, são os cuidados dos cateteres pelos profissionais de saúde que têm importância fundamental na prevenção de infecções e no prolongamento do tempo de permanência destes cateteres ${ }^{7}$.

Frente à preocupação com o controle das infecções nestes pacientes, passamos a redobrar os cuidados assépticos, protegendo sempre as conexões com gaze embebida em solução anti-séptica ${ }^{11,28}$.

As culturas sangüíneas, que antes eram feitas a cada início e término de infusão de nutrição parenteral, passaram a ser feitas somente quando havia febre ou outro sinal de infecção. O objetivo foi diminuir a manipulação do cateter e evitar aspirar sangue, procedimento que favorece a obstrução do cateter e a formação de trombos, os quais desencadeiam o crescimento de microorganismos.

A orientação dos pacientes para cuidar melhor da sua higiene, principalmente das mãos, tem sido reforçada. Mudou-se a técnica de fixação do microfusor, de maneira a evitar que a agulha se deslocasse durante a infusão de nutrição parenteral e houvesse infiltração subcutânea, necessitando uma excessiva manipulação do cateter para conseguir uma nova punção.

Concluiu-se que o protocolo de cuidados de cateteres venosos centrais permanentes é essencial para a prevenção de infecção e para o prolongamento do tempo de uso destes cateteres, possibilitando a continuidade da oferta de nutrientes essenciais à vida. Os pacientes em uso destes cateteres, em sepsis bacteriana, podem ser tratados e seus cateteres podem ser mantidos.

\section{REFERÊ NCIAS}

1. Brothers $T$, von Moll LK, Niederhuber JE, Roberts JA, Walker-Andrews S, Ensminger WD. Experience with subcutaneous infusion ports in three hundred patients. Surg Gynecol Obstet. 1988; 166(4): 295-301.

2. Niederhuber JE, Ensminger WG, Gyves JW, Liepman M, Dora K, Cozzi E. Totally implanted venous and arterial access system to replace external catheters in cancer treatment. Surgery. 1982; 92:706-12.

3. Vanek VW. The ins and outs of venous access: Part II. Nutr Clin Pract. 2002; 17(3):142-55.

4. Ryder M. The future of vascular access: Will the benefits be worth the risk? Nutr Clin Pract. 1999; 14:165-9.

5. Eastridge BJ, Lefor AT. Complications of indwelling venous access devices in cancer patients. J Clin Oncol. 1995; 13(1):233-8.

6. Groeger JS, Lucas AB, Thaler HT, Friedeander-klar $H$, Brown $A E$, Kiehn TE, et al. Infectious morbidity associated with long-term use of venous access devices in patients with cancer. Ann Intern Med. 1993; 119(12):1168-74.

7. Severien C, Nelson JD. Frequency of infections associated with implanted systems vs cuffed, tunneled Silastic venous catheters in patients with acute leukemia. Am J Dis Child. 1991; 145(12): 1433-8.

8. Sotir MJ, Lewis C, Bisher EW, Ray SM, Sourcie JM, Blumberg HM. Epidemiology of device-associated infections related to a long-term implantable vascular access device. Infect Control Hosp Epidemiol. 1999; 20(3):187-91. 
9. Sweed MR, Guenter P, Jones S. Nursing implications for the adult patient receiving nutritional support. Medsurg Nurs. 1995; 4(2):99-107.

10. Vanek VW. The ins and outs of venous access: Part I. Nutr Clin Pract. 2002; 17(2):85-98.

11. Wurzel CL, Halom K, Feldman JG, Rubin LG. Infection rates of Broviac-Hickman catheters and implantable venous devices. Am J Dis Child. 1988; 142(5):536-40.

12. Kock HJ, Kranse U, Pietsch M, Rasfeld S, Walz MK. Implantable catheter systems. Experiences with 1000 patients with central venous ports. Dtsch Med Wochenschr. 1996; 121(3):47-51.

13. Dinc LE. The effectiveness of an educational intervention in changing nursing practice, and preventing catheter-related infection for patients receiving total parenteral nutrition. Int J Nurs Stud. 2000; 37:371-3.

14. Krzywda EA, Andris DA, Edmiston CE Jr. Catheter infections: diagnosis, etiology, treatment, and prevention. Nutr Clin Pract. 1999; 14:178-90.

15. Schwank S, Rajacic Z, Zimmerli W, Blaser J. Impact of bacterial biofilm formation on in-vitro and in vivo activities of antibiotics. Antimicrob Agents Chemother. 1998; 42:895-8.

16. Byers $K$, et al. Case fatality rate for catheter-related bloodstream infections: A meta-analysis. Proceedings of the 5th annual meeting of the Society for Hospital Epidemiology of America. San Diego, CA, April 2-4, 1995.

17. Stillman RM, Soliman F, Garcia L, Sawyer PN. Etiology of catheter-associated sepsis. Correlation with trombogenicity. Arch Surg. 1977; 112(12): 1497-9.

18. Raad II, Hanna HA. Intravascular catheter-related infections: new horizons and recent advances. Arch Intern Med. 2002; 162(8):871-8.

19. Raad II, Luana M, Khalil SA, Casterton JW, Lam C, Bodey GP. The relationship between the thrombotic and infectious complications of central venous catheters. JAMA. 1994; 271:1014-6.

20. Timsit JF, Farkas JC, Boyer JM, Martin JB, Misset B, Renaud B, et al. Central vein catheter-related thrombosis in intensive care patients: Incidence, risks factors, and relationship with catheter-related sepsis. Chest. 1998; 114(1):207-13.

21. Unamuno MRDL, et al. Programa de assistência nutricional a pacientes portadores de síndrome do intestino curto. Anais do 11을 Congresso da Sociedade Brasileira de Nutrição Parenteral e Enteral; 1995, out; Caxambu. Caxambu: SBNC; 1995.

22. Marchini JS, et al. Três anos de tratamento e seguimento de pacientes portadores de síndrome de má absorção grave, portadores de desnutrição refratária à dietoterapia via oral. Rev Bras Nutr Clín. 1996; 11:27-42.

23. Marchini JS, et al. Síndrome do Intestino Curto: dificuldades de tratamento, sobrevida e evolução clínico-nutricional de longo prazo. Rev Col Bras Cir. 1995; Supl 2:167.

24. Unamuno MRDL, et al. Experiencia de cuatro años com el catéter venoso permanente totalmente implantable para nutrición parenteral. Anais do 6ㅇ Congresso Latino Americano de Cirurgia, 2은 Congresso Chileno de Nutrición Clínica; 1997 ago.

25. Unamuno MRDL, et al. Estudo microbiológico em cateteres venosos totalmente implantados usados para nutrição parenteral nos pacientes da Unidade Metabólica do HCFMRP. Anais do 12 Congresso da Sociedade Brasileira de Nutrição Parenteral e Enteral; 1997 nov; São Paulo. São Paulo: SBNC; 1997.

26. Halpin DP, O'Byrne P, McEntee G, Hennessy TP, Stephens RB. Effect of a betadine connection shield on central venous catheter sepsis. Nutrition. 1991; 7(1):33-4.

27. Sitges-Serra A. Strategies for prevention of catheterrelated bloodstream infections. Support Care Cancer. 1999; 7(6):391-5.

28. Faubion WC, Wesley JR, Khalidi N, Silva J. Total parenteral nutrition catheter sepsis: Impact of the team approach. JPEN. 1986; 10(6):642-5.

29. Brasil. Secretaria de Vigilância Sanitária do Ministério da Saúde; Portaria 272 abr 81998. Brasília; 1998.

30. Caressia AC, et al. Síndrome de Intestino Curto (SIC): ingestão alimentar domiciliar de pacientes acompanhados no ambulatório da Unidade Metabólica de Nutrição Clínica do Hospital das Clínicas de Ribeirão Preto. Anais do 3ํㅡㄹ Congresso Brasileiro de Clínica Médica; 1995 out; São Paulo. São Paulo: SBCM; 1995.

31. Marchini JS, et al. Suporte Nutricional de longa duração em pacientes, após retirada de mais de dois metros de intestino delgado. Anais do $21 \%$ Congresso Brasileiro de Cirurgia, 11 Congresso Latino Americano de Cirurgia; 1995 set; São Paulo. São Paulo: SBC; 1995.

32. Marchini JS. Síndrome do intestino curto: aspectos fisiopatológicos, clínicos e suporte nutricional de longa duração. Rev Med. 1994; 27(3/4):310-9.

33. Nonino CB. Terapia nutricional em pacientes portadores de síndrome de má absorção grave. Anais do 12ำ Congresso da Sociedade Brasileira de Nutrição Parenteral e Enteral; 1997 nov; São Paulo. São Paulo: SBNC; 1997.

34. Unamuno MRDL, et al. Complicação infecciosa relacionada a cateter venoso permanente, em uma 
paciente recebendo nutrição parenteral. Anais do 7으 Congreso Latino Americano de Nutrición Parenteral y Enteral, 2ㅇ Conferencia Centroamericana Y del Caribe de Nutrición Clínica; 1999 ago.

35. Pittet D, Tarara D, Wenzel RP. Nosocomial bloodstream infection in critically ill patients: excess length of stay, extra-costs and attributable mortality. JAMA. 1994; 272:1598-601.

36. Guédon C, Nouvellon M, Lalaude O, Larebours E. Efficacy of antibiotic-lock technique with teicoplamin in staphylococcus epidermidis catheter-related sepsis during long-term parenteral nutrition. JPEN. 2002; 26(2):109-13.

37. Brandão MR, et al. Cateter totalmente implantável em pacientes oncológicos. Experiência de 46.076 dias. Rev Bras Nutr Clín. 1997; 12:66-72.

38. Schmidt-Sommerfeld E. Catheter-related complications in 35 children and adolescents with gastrointestinal disease, on home parenteral nutrition. JPEN. 1990; 14(2):148-53.

39. Mueller BU, Skelton J, Callender DP, Marshall D, Gress J, Longo D, et al. A prospective randomized trial comparing the infectious and non-infectious complications of an externalized catheter versus a subcutaneously implanted device in cancer patients. J Clin Oncol. 1992; 10(12):1943-8.
40. Young GP, Alexeyeff M, Russel DM, Thomas RJ. Catheter sepsis during parenteral nutrition: The safety of long-term opsite dressings. JPEN. 1988; 12(4):365-70.

41. Cuntz D, Michaud L, Guimber D, Husson MO, Gottrand F, Turck D. Local antibiotic lock for the treatment of infections related to central catheters in parenteral nutrition in children. JPEN. 2002; 26(2):104-8.

42. Issam RI, Hend $\mathrm{H}$. Intravascular catheter related infections: new horizons and recent advances. Arch Intern Med. 2002; 162:871-8.

43. O'keefe SJD, Burnes JU, Thompson RL. Recurrent sepsis in home parenteral nutrition patients: an analysis of risk factors. JPEN. 1994; 18(3):256-63.

44. Ma TY, Yoshinaka R, Banaag A, Johnson B, Davis S, Berman SM. Total parenteral nutrition via multilumen catheter does not increase the risk of catheter-related sepsis: a randomized, prospective study. Clin Infec Dis. 1998; 27(3):500-3.

45. Eyer S, et al. Catheter related sepsis: prospective, randomized study of three methods of long-term catheter maintenance. Crit Care Med. 1990; 18(10):1073-9.

Recebido para publicação em 8 de agosto de 2003 e aceito em 18 de março de 2004. 